\title{
Solid-Phase Microextraction Fiber Coating for Biological Analysis
}

\author{
Yi Yu ${ }^{1,2}, \mathrm{Xu} \mathrm{Fei}^{1, \mathrm{a}}$, Jing Tian ${ }^{2, \mathrm{~b}}$, Longquan $\mathrm{Xu}^{1}$, Yi Wang ${ }^{2}$ \\ ${ }^{1}$ Instrumental Analysis Center, Dalian Polytechnic University, Dalian, 116034, China \\ ${ }^{2}$ School of Biological Engineering, Dalian Polytechnic University, Dalian, 116034, China \\ aemail: feixu@dlpu.edu.cn, bemail: tianjing@dlpu.edu.cn
}

Keywords: solid-phase microextraction; biological molecule; soyasaponin.

\begin{abstract}
A procedure for solid-phase microextraction fiber preparation was introduced, which combined a quartz fiber substrate with a blend of $\mathrm{SiO}_{2}$ sol-gel and PVA, as a way of increasing both the adhesive ability and the extracting capability of fibers. Double cross-linked network of organic and inorganic layer was prepared for the fiber coatings by repeated freezing-thawing. The effects of salt on fiber coating composition and microextraction conditions were investigated. The relative standard deviations (RSD) $(n=3)$ were obtained between $6.9 \%$ and $10.2 \%$. The fiber coatings for biological molecule's extraction of soyasaponin were presented.
\end{abstract}

\section{Introduction}

Solid-phase microextraction (SPME) was first introduced by Pawliszyn and co-workers in 1990s [1]. In recent years, as a sample preparation technique it has been widely used in many fields including the analysis of environment, food, natural products, toxicology, biology and forensic samples [2][3]. This technique has important advantages over conventional extraction techniques due to its ease of use and being rapid, portable, and solvent- free [4]. The key SPME technique is the sorbent coating immobilized on the fiber substrate [5]. Development of new fiber coatings has been deemed as a major challenge for enhancement of sensitivity and selectivity [6].Poly(vinyl alcohol) (PVA) is a semi-crystalline synthetic polymer which can form physically cross-linked hydrogels by repeatedly freezing and thawing PVA aqueous solutions [7][8]. It has attracted much attention for their potential applications in biomedical materials [9], drug delivery [10], biotechnology and cell immobilization [11]. PVA hydrogels obtained via this method show many appealing properties such as high mechanical strength, rubber-like elasticity, ability to retain their original shape, biocompatibility, and lack of toxicity [6][9][10], which make it a very suitable coating material for SPME.However, most organic coatings are difficult to be attached to the silicon dioxide substrate. To improve the adhesion performance, sol-gel has been introduced as the coating material which also demonstrates high porosity and large surface area due to its polymeric network. In addition, it expresses high thermal stability and chemical stability.

In this paper, innovative solid-phase microextraction coatings with double cross-linked network were prepared by sol-gel technology and repeated freezing-thawing. The coatings have high thermal stability, good solvent resistance, satisfactory coating preparation repeatability and long lifetime. All these advantages make it an ideal candidate for the analysis of biological molecules.

\section{Preparation of SPME fiber}

The fibers ( $\sim \mathrm{cm}$, length) were treated by an etchant consisting of $\mathrm{H}_{2} \mathrm{SO}_{4} / \mathrm{H}_{2} \mathrm{O}_{2}(7: 3 \mathrm{v} / \mathrm{v})$ at $90 \mathrm{C}$ for $1 \mathrm{~h}$ to remove the polyimide layer and expose the fused silica core followed by a rinse with deionized water and then dried using nitrogen gas.The $\mathrm{PVA} / \mathrm{SiO}_{2}$ sol was prepared as follows: PVA $(2 \mathrm{~g})$ was dissolved in deionized water $(40 \mathrm{~mL})$ at $90 \mathrm{C}$ under stirring and refluxed for $2 \mathrm{~h}$ to form a homogeneous PVA solution (5.0 wt.\%). The mixture of TEOS (18.5 mL), ethanol (19 mL), deionized water $(9 \mathrm{~mL})$ and $\mathrm{HCl}(2 \mathrm{~mL})$ was stirred for $2 \mathrm{~h}$ at room temperature to get a $\mathrm{SiO}_{2}$ sol. Then, the formed $\mathrm{SiO}_{2}$ sol was slowly added to PVA solution and the mixture was stirred for $4 \mathrm{~h}$ at 
$50 \mathrm{C}$ to obtain a transparent $\mathrm{PVA} / \mathrm{SiO}_{2}$ sol. The treated fibers were dipped into the $\mathrm{PVA} / \mathrm{SiO}_{2}$ sol for $30 \mathrm{~min}$, then taken out vertically, frozen at $-20 \mathrm{C}$ for $6 \mathrm{~h}$ and thawed at room temperature for $2 \mathrm{~h}$. For each fiber this coating process was repeated six times to obtain the targeted thickness. Then, the coated SPME fibers were rinsed by deionized water and ethanol to remove acid. Finally, the prepared fibers were dried in freeze dryer at $-50 \mathrm{C}$ for $12 \mathrm{~h}$ and in oven at $120 \mathrm{C}$ for $12 \mathrm{~h}$.

\section{SPME procedure}

The extraction was performed by placing fibers coated with $\mathrm{PVA} / \mathrm{SiO}_{2}$ into the working solution, which was stirred at a constant speed with a magnetic stirrer for $5 \mathrm{~min}$ at $25 \mathrm{C}$. After extraction, the fibers were inserted into a $5 \mathrm{~mL}$ of vial containing $2 \mathrm{~mL}$ methanol to desorb sugars, and the desorption solvent for soyasaponin was $2 \mathrm{~mL}$ mobile phase solution of HPLC. The extracted sugars and soyasaponin were detected by UV-Vis spectrophotometry and HPLC analysis, respectively. Once an extraction-desorption cycle was completed, the fiber was immediately rinsed by ethanol and dried in oven at $80 \mathrm{C}$ to preserve the porous structure.

\section{Test results}

\section{Fiber coating preparation and characterization}

The morphology of $\mathrm{PVA} / \mathrm{SiO}_{2}$ fibers was investigated by scanning electron microscopy (SEM) under different magnifications (Figure 1A), showing a homogeneous and porous coating on the entire fiber surface. It also presented that there were interpenetrating double cross-linked networks between PVA hydrogel and $\mathrm{SiO}_{2}$. The thickness of the developed coatings was calculated to be about $20 \mu \mathrm{m}$ (Figure $1 \mathrm{~B}$ shows the bare fiber). Higher thicknesses can be obtained by increasing the number of dipping times. The porous structure of the fiber surface provides a high surface area and inner space enhancing the extraction capacity in the SPME process.

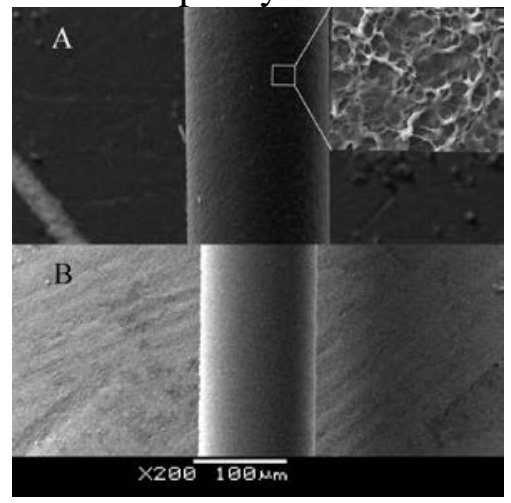

Figure 1. Scanning electron micrographs of fibers: (A) with the sol-gel coated PVA coating and (B) without coating.

\section{Sugar Extration}

Generally, the addition of salt (sodium chloride) increases the ionic strength in aqueous samples, decreases the solubility of polar compounds, and enhances the amount of analyte extracted by "salting out" effect. As illustrated in Figure 2, the effect of salt was investigated by adding different concentration of $\mathrm{NaCl}$ to sugar solutions ranging from 0 to $0.25 \mathrm{gmL}^{-1}$. The results showed the extraction efficiency significantly decreased with the increase of $\mathrm{NaCl}$ concentration for glucose and sucrose. For $\beta$-glucan, the extraction reached the highest with $0.05 \mathrm{gmL}^{-1}$ sodium chloride but then decreased with higher $\mathrm{NaCl}$ concentration. It can be concluded that no sodium chloride or low concentration was more suitable for extracting sugars. 


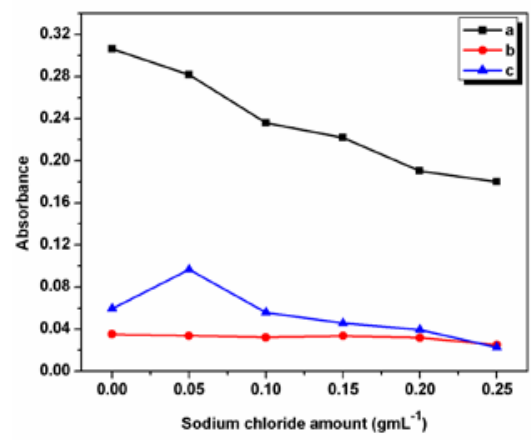

Figure 2. Absorbance as a function of salt concentration (a) glucose; (b) sucrose; (c) oat $\beta$-glucan.

The reproducibility of fibers was tested by using a single fiber and several different fibers for extracting sugars. Table 1 showed the relative standard deviation (RSD, $\mathrm{n}=3$ ) of sugars was $10.2 \%$, $6.9 \%$ and $8.4 \%$ respectively when using one fiber to perform the extraction. Another experiment with three different fibers illustrated the relative standard deviation (RSD, $n=3$ ) was less than $15 \%$ for sugars. The above analyses demonstrated that this extraction method was repeatable.

Table 1 Calibration results and precision for extraction of sugars

\begin{tabular}{llcc}
\hline Compounds & Calibration equation & Correlation coefficient, $\mathrm{R}^{2}$ & $\mathrm{RSD}(\%, \mathrm{n}=3)$ \\
\hline Glucose & $\mathrm{y}=50.543 \mathrm{x}+0.076$ & 0.9911 & 10.2 \\
Sucrose & $\mathrm{y}=6.571 \mathrm{x}-0.005$ & 0.9970 & 6.9 \\
$\beta$-Glucan & $\mathrm{y}=33.217 \mathrm{x}+0.0027$ & 0.9975 & 8.4 \\
\hline
\end{tabular}

\section{Extraction of soyasaponins}

Extraction conditions were as follows: extraction at $25 \mathrm{C}$ for $30 \mathrm{~min}$, desorption at $25 \mathrm{C}$ for 20 min, constant stirring without $\mathrm{NaCl}$, and the fiber coating with $\mathrm{SiO}_{2}$ content of $20 \mathrm{wt}$.\%. Figure 3 was a comparison chromatogram between direct injection of soyasaponin (Figure 3 a) and extraction using sol-gel-coated PVA fibers (Figure $3 \mathrm{~b}$ ). The retention times at 1, 2, 3 represented group B soyasaponins V, IV and II, respectively. The chromatogram of extraction demonstrated that the sol-gel-coated PVA fibers have relatively strong capability of adsorbing soyasaponins.

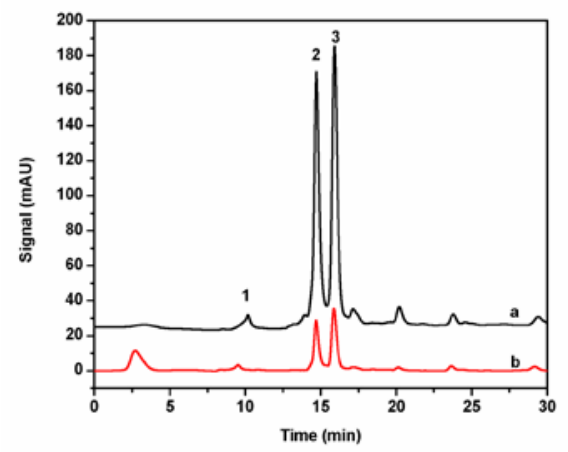

Figure 3. Chromatograms of group B soyasaponins obtained at $205 \mathrm{~nm}$ by direct injection (a) and extraction (b) using the sol-gel coated PVA fiber. Peaks: 1. soyasaponins soyasaponins II.

\section{Conclusion}

In this study, a new SPME coating from PVA/SiO ${ }_{2}$ composite with double cross-linked network based on quartz fiber was introduced. The sol-gel coated PVA coating provided a porous structure and large surface area that accelerated extraction, mass transfer rates and enhanced extraction efficiency. The fibers were successfully employed to extract polar sugars and group B 
soyasaponins with a strong capability, and the low RSD values demonstrated precision and reliability of the fibers.

\section{Acknowledgement}

This work was supported by National Natural Science Foundation of China (No.21204007), Excellent talents support program of colleges and universities of liaoning province (LJQ2014054), Natural Science Foundation of Liaoning Province of China (2013020167), Open Project of State Key Laboratory for Supramolecular Structure and Materials (SKLSSM201425).

\section{References}

[1] Catherine L Arthur, Janusz Pawliszyn. Solid Phase Microextraction with Thermal Desorption Using Fused Silica Optical Fibers [J]. Analytical Chemistry, 199062 (19) 2145-2148.

[2] Ruifen Jiang, Fang Zhu, Tiangang Luan, Yexiang Tong, Hong Liu, Gangfeng Ouyang, Janusz Pawliszyn. Carbon nanotube-coated solid-phase microextraction metal fiber based on sol-gel technique [J]. Journal of Chromatography. A, 20091216 (22) 4641-4647.

[3] Mihaela L. Musteata, Florin Marcel Musteata, Janusz Pawliszyn. Biocompatible Solid-Phase Microextraction Coatings Based on Polyacrylonitrile and Solid-Phase Extraction Phases [J]. Analytical Chemistry, 200779 (18) 6903-6911.

[4] Mohammad Bagher Gholivand, Mir Mahdi Abolghasemi, Peyman Fattahpour. Polypyrrole/hexagonally ordered silica nanocomposite as a novel fiber coating for solid-phase microextraction [J]. Analytica Chimica Acta, 2011704 174-179.

[5] Xin Zhou, Pengfei Xie, Jiang Wang, Beibei Zhang, Mingming Liu, Hanlan Liu, Xionghan Feng. Preparation and characterization of novel crown ether functionalized ionic liquid-based solid-phase microextraction coatings by sol-gel technology [J]. Journal of Chromatography A, 20111218 (23) 3571-3580.

[6] Habib Bagheri, Ali Aghakhani, Masoud Baghernejad, Alireza Akbarinejad. Novel polyamide-based nanofibers prepared by electrospinning technique for headspace solid-phase microextraction of phenol and chlorophenols from environmental samples [J]. Analytica Chimica Acta, 2012716 34-39.

[7] Rosa Ricciardi, Finizia Auriemma, Claudio De Rosa, Francoise Laupretre. X-ray diffraction analysis of poly(vinyl alcohol) hydrogels, obtained by freezing and thawing techniques [J]. Macromolecules, 200437 (5) 1921-1927.

[8] Nautiyal Arvind, Sekhar K C, Pathak N P, Nath R. Ferroelectric and phase transition studies in cesium nitrate: poly(vinyl alcohol) composite films [J]. Applied Physics Section A: Materials Science and Processing, 200997 (1) 205-210.

[9] Thompson R, Creavin A, O'Connell M, O'Connor B, Clarke P. Optimization of the enzyme-linked lectin assay for enhanced glycoprotein and glycoconjugate analysis [J]. Analytical Biochemistry: Methods in the Biological Sciences, 2011413 114-122.

[10] Christie M. Hassan, Nikolaos A. Peppas. Structure and Applications of Poly(vinyl alcohol) Hydrogels Produced by Conventional Crosslinking or by Freezing/Thawing Methods [J]. Advances in Polymer Science, 2000153 37-65.

[11] Lozinsky VI, Galaev IY, Plieva FM, Savinal IN, Jungvid H, Mattiasson. Polymeric cryogels as promising materials of biotechnological interest [J]. Trends in biotechnology, 200321 (10) 445-451. 